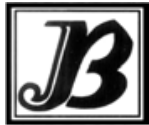

J. bio-sci. 15: 7-13, 2007

ISSN 1023-8654

http://www.banglajol.info/index.php/JBS/index

\title{
CYTOLOGICAL STUDIES OF NYMPHAEA SPECIES AVAILABLE IN BANGLADESH
}

\author{
A Hossain, G Kabir ${ }^{1}$, M M Ud-deen ${ }^{*}$ and A M S Alam ${ }^{3}$ \\ Professor Sultanul Alam Cytogenetics Laboratory, \\ Department of Botany, University of Rajshahi, Rajshahi-6205, Bangladesh \\ ${ }^{1}$ Department of Botany, ${ }^{2}$ Department of Crop Science \& Technology \\ ${ }^{3}$ Department of Agronomy and Agricultural Extension, University of Rajshahi, Rajshahi
}

\begin{abstract}
The present investigation was undertaken in order to study the somatic karyotypes of four species of Nymphaea available in Bangladesh. Karyotypes of four species of Nymphaea revealed that most of the chromosomes were metacentric indicating their primitive nature and chromosome counts revealed a polyploid series of tetraploid, pentaploid and hexaploid based on $X=14$. Findings of the present study indicated that Nymphaea pubescens is a hexaploid ( $6 \mathrm{X}=84$, white flower) which might have originated by chromosome doubling of Nymphaea daubeniana $(3 X=42)$ while Nymphaea rubra and Nymphaea nouchali (Type-1) are tetraploid $(4 X=56)$ species.
\end{abstract}

Key words: Nymphaea, karyotype

\section{Introduction}

Nymphaea species are aquatic herbs with perennial rhizomes or rootstocks anchored with mud. In Bangladesh, the common Bengali name of Nymphaea is "Shapla". In addition to many natural hybrids, a large number of artificially raised varieties (Gray 1900, Ames 1900 and Nult 1967) have been increasing the list of Nymphaeas. Gupta (1980) investigated the genus Nymphaea cytologically and commented on their evolutionary relationship. His findings revealed a polyploid series ranging from diploid to octaploid levels based on $X=14$. He had noticed various abnormalities in the mitotic and meiotic configurations. Nymphaea species with white flower is the national flower of Bangladesh. Cytomorphological report on this species along with other species is very much scanty in the country. The present study was thus undertaken on six different types of Nymphaea available in Bangladesh from cytomorphological point of view.

\section{Materials and methods}

Four species of Nymphaea comprising six different types were used as plant material in this study. The species were Nymphaea pubescens, Nymphaea alba (it might be cultivated somewhere else, but it was found to be grown wild in different places at Rajshahi, Bangladesh from where it was used as experimented material), Nymphaea rubra and Nymphaea nouchali (Type 1,2 and 3) and they were collected from different parts of Rajshahi district. A thorough morphological study was made on these species (Table 1). They were then differentiated into species and types with the help of available literature and the data obtained from this study. To count the chromosome number as well as to study chromosome morphology the root tips of different species were fixed 1:3 acetoalcohol and preserved in 70\% ethanol just after pre-treatment with the saturated solution of para-dichlorobenzene (PDB). Chromosome staining was performed following the schedule of Haque et al. (1976). Photomicrographs were made from the metaphase plates and the chromosomes were measured from cameralucida drawings.

\footnotetext{
*Corresponding author: Mobile: +8801558340438, Email: muddeen05@yahoo.com
} 


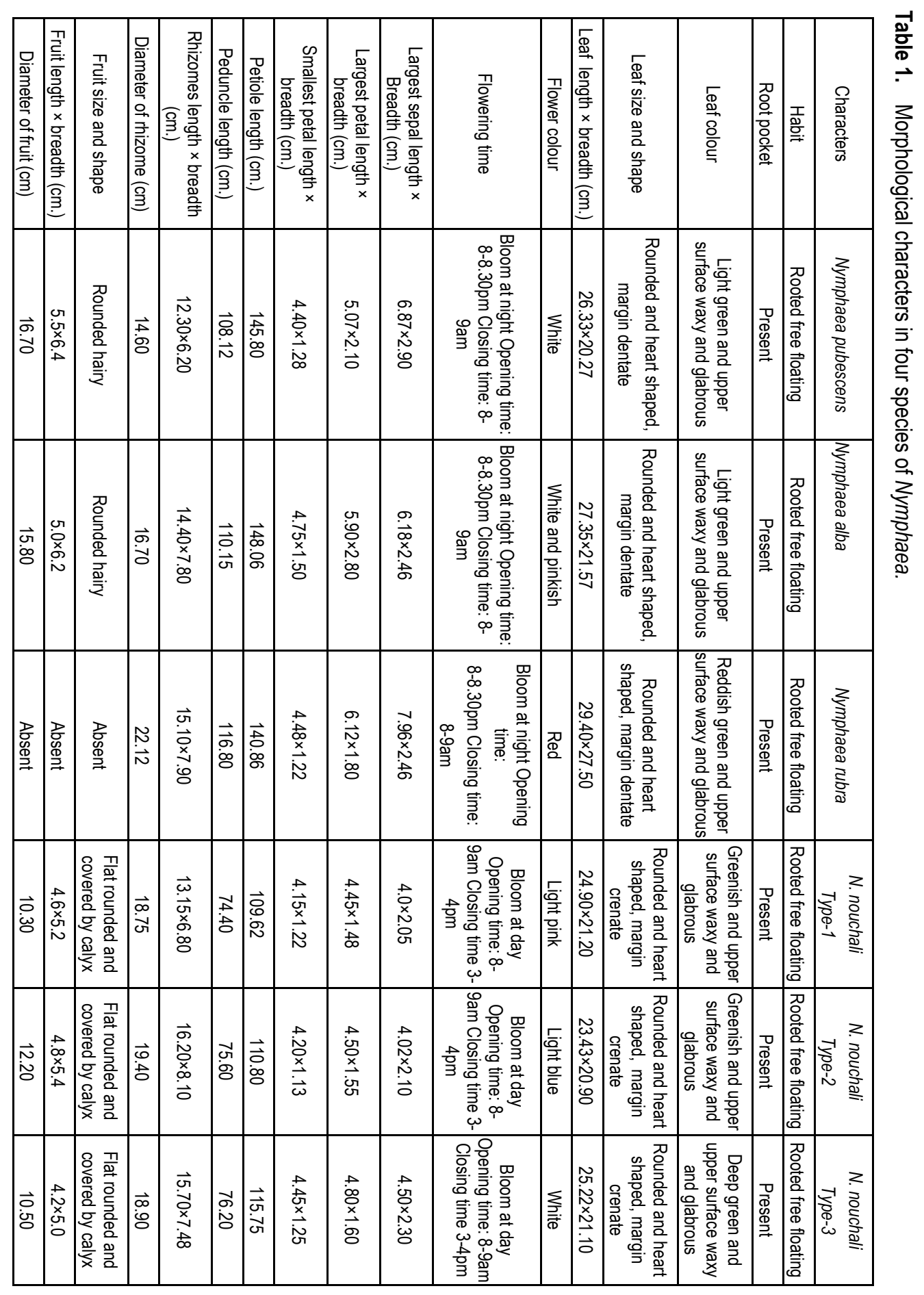




\section{Results and discussion}

A thorough morphological study was made on four species of Nymphaea. The results of this study are presented in Table 1. The microphotographs of metaphase plates of four species of Nymphaea are presented in Figures 1a-6a and the Camera Lucida drawings used for measuring the chromosome are also shown in Figures
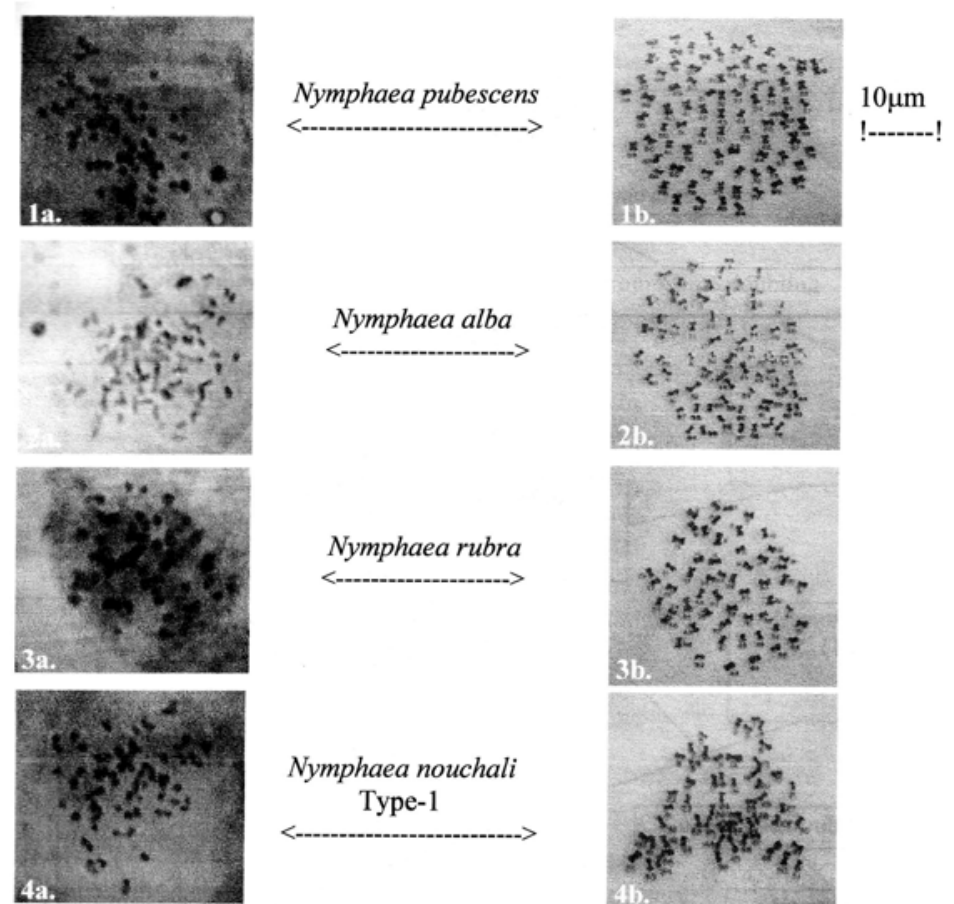

Nymphaea nouchali

Type-1
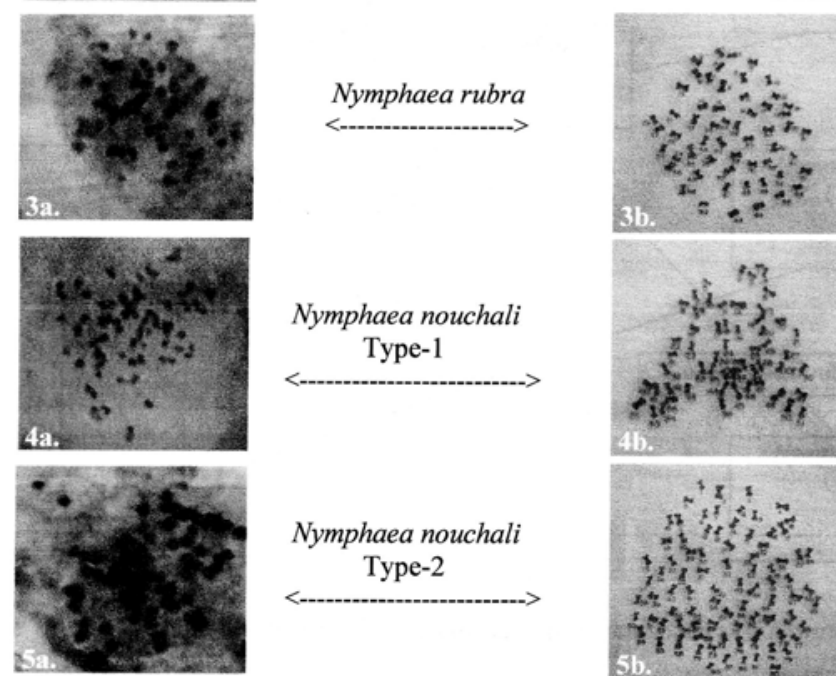

\section{Nymphaea nouchali \\ Type-2}

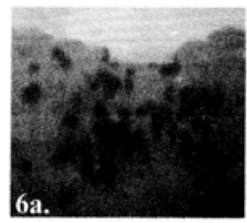

Nymphaea nouchali

Type-3
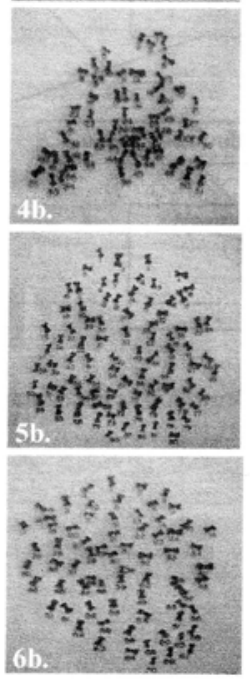

Figs. 1-6(a-b). Somatic metaphase chromosomes in six taxa of Nymphaea (Ca. 750x). 1a-6a. Photomicrographs of somatic metaphase chromosomes in six taxa of Nymphaea; $1 \mathrm{~b}-6 \mathrm{~b}$. Cameralucida drawings of somatic metaphase chromosomes in four species of Nymphaea. 
The average value $(\bar{X})$ for chromosome length, total chromatin length, total frequency, arm ratios, etc. are given in Table-2. In Nymphaea pubescens, Nymphaea alba, Nymphaea nouchali (Type 1, 2 and 3) and Nymphaea rubra the longest mean absolute lengths of chromosome sets were $3.43,2.97,2.06,3.99,3.02, \& 3.38 \mu \mathrm{m}$ and shortest mean lengths of chromosome sets were $2.34,1.44,1.56,1.82,1.70$ and $1.44 \mu \mathrm{m}$, while total chromatin lengths were $115.26,71.26,72.66,117.06,86.86 \& 66.48 \mu \mathrm{m}$, respectively. In all of the Nymphaea species TF \% were found to be the same.

Out of nearly 40 species belonging to this genus, 22 species have been investigated cytologically so far and revealed a basic number of $X=14$. Similarly euploid forms have been reported by Lohammar (1942) in Nymphaea candida, Love and Love (1942) and Ehrenberg (1945) in Nymphaea alba while Wood (1959) recorted aneuploidy in Nymphaea tetragona. In the present study the chromosome number of all the specimens were found to be euploidy in nature, viz. Nymphaea pubescens had 2n=84, Nymphaea alba had 2n=70, Nymphaea rubra had $2 n=56$, Nymphaea nouchali (Type-1) had 2n=56, Nymphaea nouchali (Type-2) had $2 n=84$, and Nymphaea nouchali (Type-3) had $2 n=70$. In the present study different chromosome number of genus Nymphaea also indicated a dominant role in speciation with basic chromosome number $X=14$. Gupta (1978) studied nineteen taxa of both Indian wild and American cultivated forms of Nymphaea and reported diploid, triploid, tetraploid and hexaploid series based on $X=14$. Gupta (1980) made the chromosome count in the genus Nymphaea and also reported diploid to octaploid based on $X=14$. Thus the present findings agreed well with the previous reports of Guignard (1898) as well as of others. However, a number of workers (Langlet and Sodenberg 1927, Lohammar 1942, Love and Love 1942, Ehrenberg 1945, Harada 1952, Heslop-Harrison 1955, Janaki Ammal 1956, Raghavan and Arora 1958, Wood 1959, Mitra and Datta 1967, Sen and Bhaduri 1971, Gupta 1972, 1976, 1978a, 1978b, 1979 and 1980) reported the six different types of Nymphaea (Figure 7), which were studied in the present investigation. However, in Nymphaea rubra 2n=56 (4x) chromosomes and Nymphaea nouchali (Type-1) 2n=56 (4x) chromosomes were found which may be evolved by the doubling of chromosome $(2 n=28)$ from ancestral species.

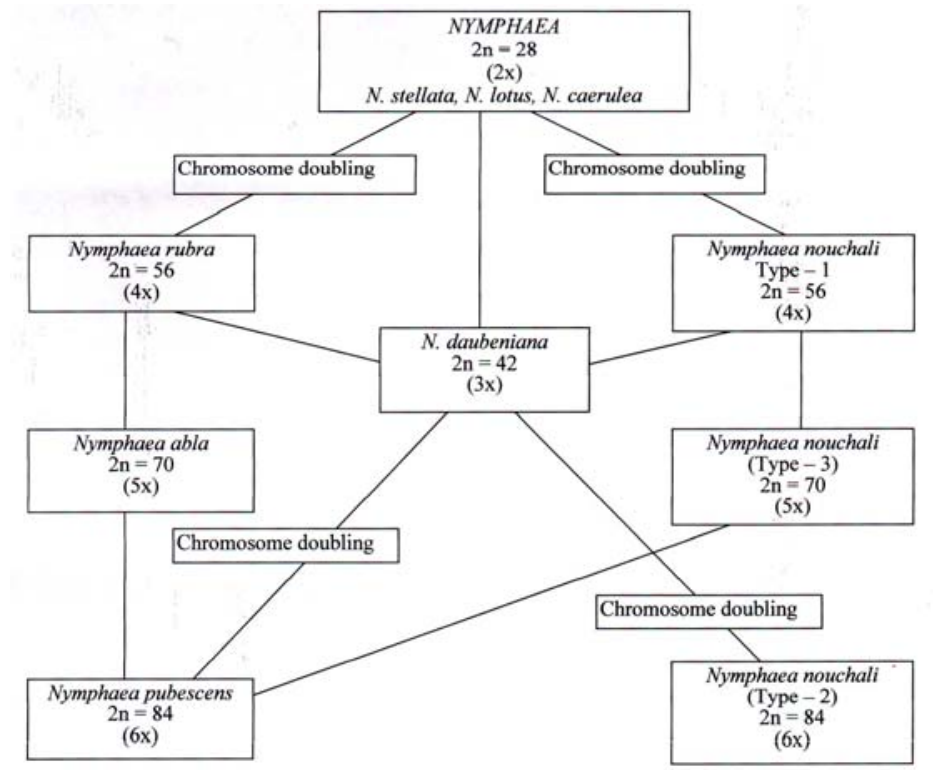

Fig. 7. Presumed phylogenetic relationships among seven species of Nymphaea based on karyotype and ploidy level. 


\begin{tabular}{|c|c|c|c|c|c|c|c|}
\hline$\leqq$ & $\leq$ & $<$ & $<$ & $\equiv$ & $=$ & - & 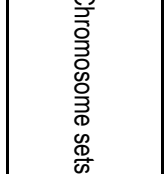 \\
\hline 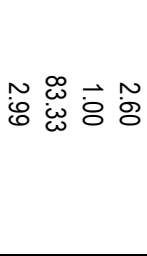 & 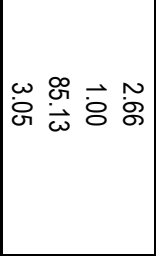 & 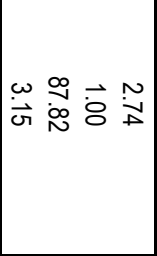 & 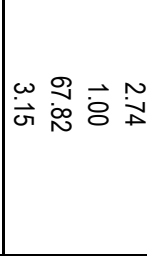 & $\mid \begin{array}{c}\infty \\
\vec{\omega} \\
\vec{\omega}\end{array}$ & 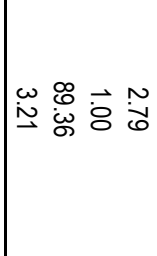 & 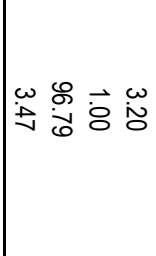 & 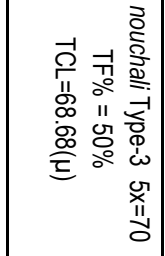 \\
\hline 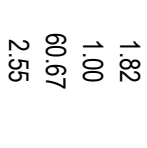 & 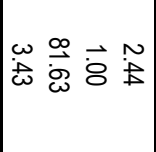 & 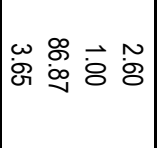 & 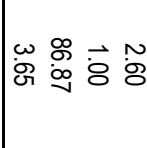 & 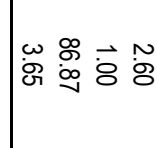 & 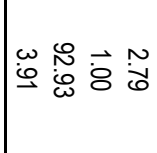 & $\overrightarrow{\dot{v}} \stackrel{\circ}{=} \overrightarrow{8}$ & 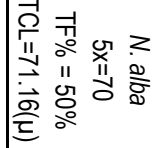 \\
\hline 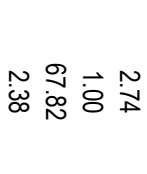 & 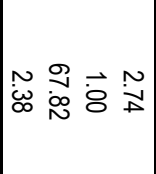 & 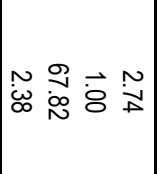 & 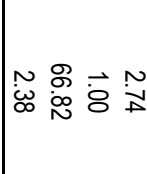 & 变过 & 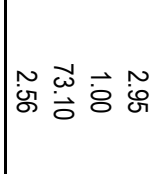 & 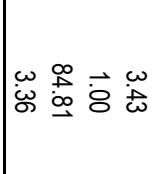 & 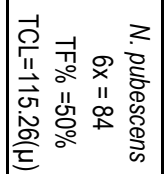 \\
\hline 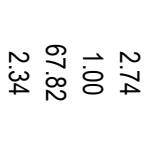 & 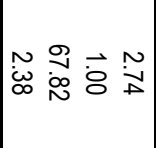 & 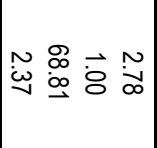 & 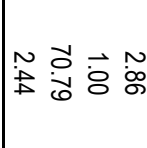 & i & 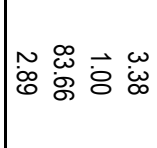 & 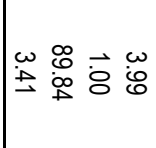 & 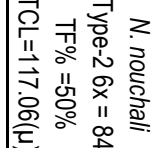 \\
\hline 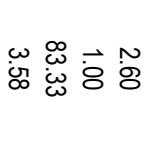 & 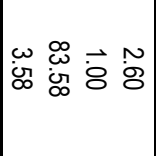 & 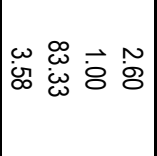 & 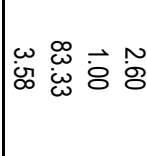 & 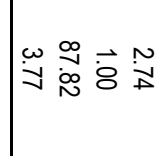 & 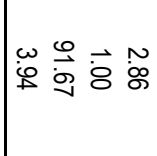 & 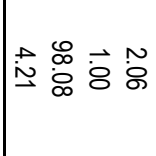 & 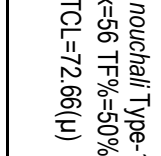 \\
\hline 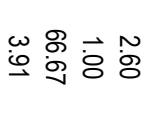 & 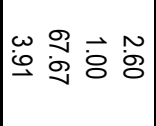 & 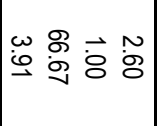 & 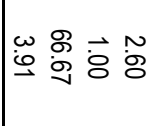 & 禹 & 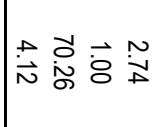 & 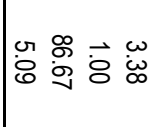 & 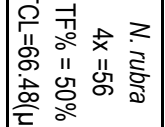 \\
\hline
\end{tabular}

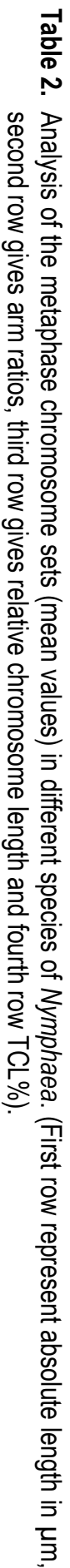




\begin{tabular}{|c|c|c|c|c|c|c|c|}
\hline$\stackrel{x}{<}$ & $\stackrel{\underline{\underline{X}}}{\underline{X}}$ & $\underline{\underline{x}}$ & $\underline{x}$ & $\times$ & $\bar{x}$ & $\leqq$ & 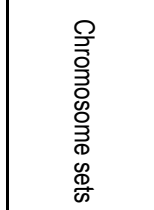 \\
\hline 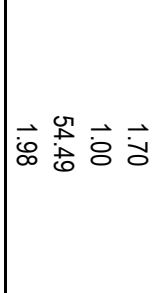 & 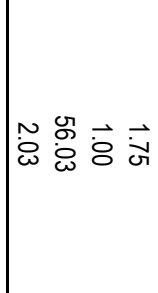 & 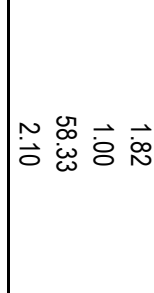 & 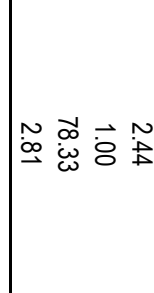 & 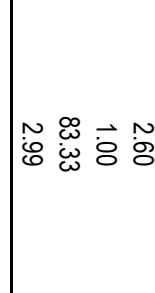 & 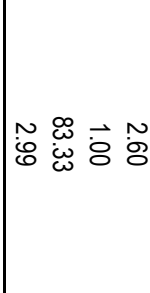 & 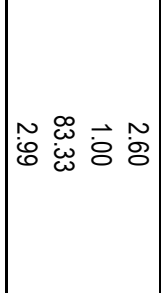 & 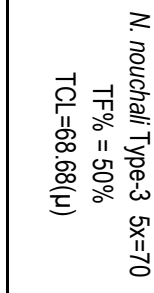 \\
\hline 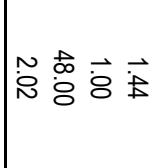 & 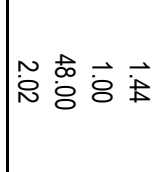 & 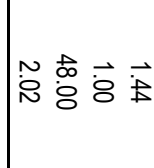 & 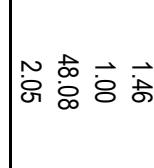 & 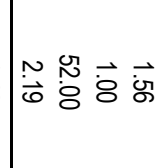 & 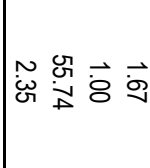 & 썽 & 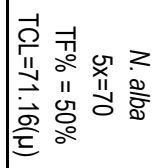 \\
\hline 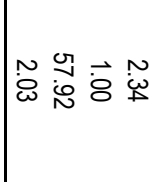 & 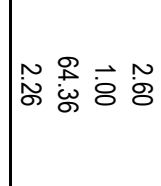 & 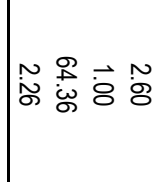 & 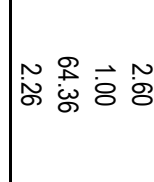 & 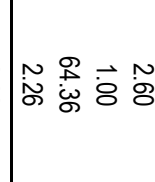 & 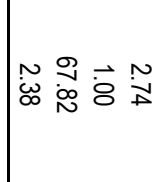 & 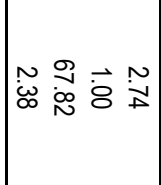 & 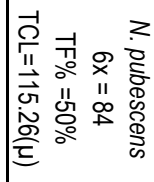 \\
\hline $\overrightarrow{\text { ஸे }}$ & 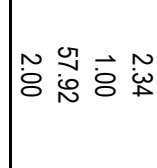 & 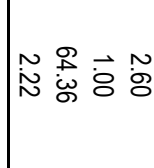 & 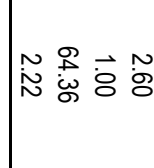 & 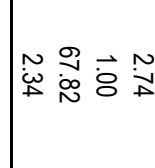 & 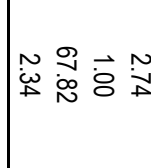 & 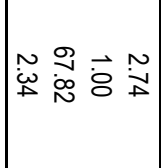 & 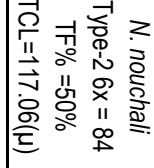 \\
\hline 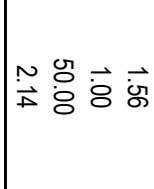 & 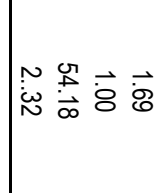 & 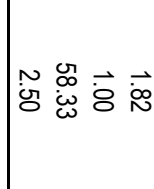 & 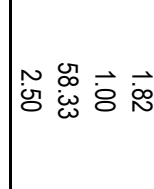 & 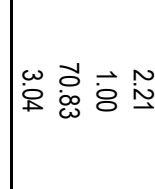 & 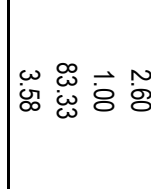 & 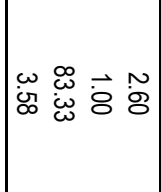 & 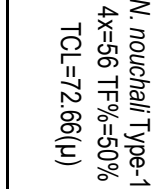 \\
\hline 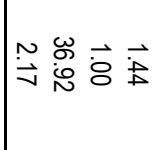 & 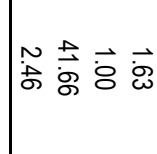 & 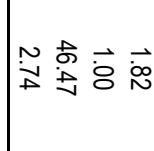 & 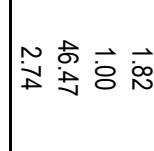 & $\mid \begin{array}{c}\omega \\
\omega \\
\omega\end{array}$ & 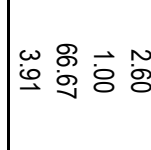 & 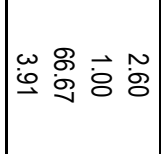 & 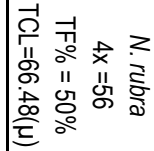 \\
\hline
\end{tabular}

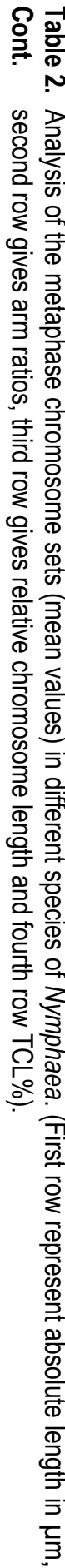


In Nymphaea daubeniana 2n=42 (3x) chromosomes were reported by Gupta (1978). This species might be evolved by the crossing of Nymphaea rubra $2 n=56$ and Nymphaea stellata or Nymphaea lotus or Nymphaea caerulea $(2 n=28)$. Nymphaea daubeniana might have also evolved by the crossing of Nymphaea nouchali (Type-1) 2n=56 (4x) and Nymphaea stellata or Nymphaea lotus or Nymphaea cerulea 2n=28. Similarly in the present study Nymphaea pubescens $2 n=84(6 x)$ and Nymphaea nouchali (Type-2) 2n=84 (6x) have been evolved by the chromosome doubling of Nymphaea daubeniana $2 n=42(3 x)$. In case of Nymphaea alba $2 n=70(5 x)$ might be appeared by the crossing of Nymphaea rubra 2n=56 (4x) and Nymphaea pubescens $2 n=84$ (6x). Likely Nymphaea nouchali (Type-3) $2 n=70$ (5x) might also be originated by the crossing of Nymphaea pubescens 2n=84 (6x) and Nymphaea nouchali (Type-1) 2n=56 (4x). None of the members in the present study was found as aneuploid. Anyhow, most of the species hybridize freely among themselves naturally. However, the cytomorphological findings in the present study indicated that Nymphaea pubescens $(6 x=84$, white flower) might have originated by chromosome doubling of Nymphaea daubeniana ( $3 x=42)$.

\section{References}

Ames $O$ (1900) An interesting group of new hybrids blooming Nymphaeas. Am. Gdng. 21: 644.

Ehrenberg L (1945) Kromosomtaleu has nagara Karvaler. Bot. Notiser. 4: 430-437.

Guignard L (1898) Les centres cinetiques chez les vegetaux. Ann/s. Sci. Nat. Botanique 8 Serie. 5: 177-220.

Gray RM (1900) New hybrid of Nymphaeas. Am. Gdng. 21: 516.

Gupta P P (1972) Meiotic studies in two hexaploid species of Nymphaea. Proc. Natl. Acad. Sci. India. 41: 16.

Gupta P P (1976) Cytogenetics of ornamentals IV. Effects of gamma irradiation on Nymphaea rubra Roxb. Cytologia. 42: 297-304.

Gupta P P (1978a) Cytogenetics of aquatic ornamentals II. Cytology of Nymphaeas. Cytologia 43: 477- 484.

Gupta P P (1978b) Chromosome number reports LXI. Taxon. 27: 386-337.

Gupta P P (1979) Cytogenetics of aquatic ornaments V. Restoration of fertility in a sterile cultivar of Nymphaea through colchioloidy. Z. Pflanzenzuchtg 82: 93-96.

Gupta P P (1980) Cytogenetics of aquatic ornaments VI. Evolutionary trends and relationship in the genus Nymphaea. Cytologia 45: 307-314.

Haque A, Ali M A, Wazuddin M and Khan M A (1976) Squash method for the mitotic chromosomes of grasses. Curr. Sci. 45(10): 382-383.

Harada I (1952) Chromosome studies of some diocotyledonous water plants. Jap. J. Genet. 27: 117-120.

Heslop- Harrison Y (1955) Nymphaea Linn. Sm. J. Ecol. 43: 719-734.

Janaki Ammal E K (1956) Central Botanical Laboratory. Bull. Bot. Surv. India. 1: 33-37.

Langlet and Soderberg E (1927) Uber die Chromosomenzahlen einiger Nymphaeaceae. Acta. Hort. Berg. 2: 85-104.

Lohammar C (1942) Chromosome studies of some diocotyledonous water plants. Jap. J. Genet. 27: 117-120.

Love A and Love D (1942) Chromosome numbers of Scandinavian plant species. Bot. Notiser. 19: 59.

Mitra K and Datta N (1967) Chromosome number reports XIII. Taxon. 16: 445- 461.

Nult P A (1967) Nymphaea x Antare; a new hybrid water lily. Am. Hort. Mg. 46: 243-244.

Raghava R S and Arora C M (1958) Chromosome number in India medicinal plants.II. Proc. Ind. Acad. Sci. 47b: 352-358-4.

Sen S and Bhaduri P N (1971) Cytotaxonomy of Nymphaeaceae. J. Cytol. Genet. 6: 67:69.

Wood C E (1959) The genera of the Nymphaeaceae and Ceratophyllaceae in the South Eastern United States. J. Ecol. 43:719-734. 